Plant Tissue Cult. \& Biotech. 23(1): 67-78, 2013 (June)

$\overline{\text { PTC\&B }}$

\title{
A Proteomic Analysis of Leaf Proteins Expressed in a Brassica Somatic Hybrid and its two Parental Species
}

\author{
M. M. Hossain ${ }^{1}$, X. Li ${ }^{2}$, I. H. Evans and M. A. Rahman* \\ Department of Life Sciences, School of Science, University of Greenwich, Chatham \\ Maitime, Medway, Kent ME4 4TB, UK \\ Key words: Brassica, Rubisco, Proteomic analysis, Somatic hybrid
}

\begin{abstract}
Cabbage (Brassica oleracea L.) is one of the major vegetable crops in the world. Several somatic hybrids between cabbage and Chinese cabbage have been produced. These show hybrid vigor. To identify differences in protein expression that might be relevant to hybrid characteristics of interest, proteins from leaves of a somatic hybrid and its two parents were subjected to 1D and 2D gel analyses, followed by MALDI-TOF and MS/MS of selected spots. 1D gel analysis of the leaf proteins at 14th day post-germination (14 DPG) showed very similar profiles for all three Brassicas. However, upon 2D gel analysis, several differentially expressed protein spots from leaves were discerned. Of the five differentially expressed leaf proteins examined, all the protein spots were photosynthesisrelated, including RbcS (the rubisco small subunit). It is possible that differential expression of these photosynthesis-related proteins may relate to the improved growth of the somatic hybrid.
\end{abstract}

\section{Introduction}

Cabbage (Brassica oleracea L. var. capitata) and Chinese cabbage (B. campestris L. var. pekinensis) are important vegetables, with world-wide use, and where propagation is entirely via seeds. In the mild winters of the tropics and subtropical regions, seed production by cabbage (Brassica oleracea L. var. capitata) is very problematic due to their vernalization process. To introduce seed vernalization characteristic into cabbage, significant numbers of somatic hybrids have been produced between cabbage (cv. Yoshin) and Chinese cabbage (cv. Kenshin) through protoplast fusion (Hossain and Yazawa 1994, Yamagishi et al. 1992). Somatic hybridization is a developing field of great importance and has

*Author for correspondence: <a.rahman@ greenwich.ac.uk>. ${ }^{1}$ Department of Horticulture, Faculty of Agriculture, BSMRAU, Gazipur-1706, Bangladesh. ${ }^{2}$ Sekisui Diagnostics, 50 Gibson Drive, Kings Hill, Kent ME19 4AF, UK. 
opened up the possibility of combining genetic factors between sexually incompatible species, thereby increasing the spectrum of genetic variation in higher plants (Yamagishi et al. 1992, Hossain and Asahira 1992, Choung et al. 1988). Interspecific somatic hybridization in Brassica has various advantages over sexual hybridization. For example, cytoplasmic organelles can be hybridized by cell fusion, and this may provide additional new genetic variations useful for future new breeding programs. However, the success in interspecific sexual hybridization is limited and it is more difficult when cabbage is used as the female parent (Hossain et al. 1988, Inomata 1997).

Somatic hybrid cabbages can produce larger seeds (average weights of 3.4, 2 and $5 \mathrm{mg}$ per seed of cabbage, Chinese cabbage and somatic hybrid, respectively) and grow faster than their parents, starting at germination, which occurs earlier than in either parent (Hossain and Asahira 1992)

These novel traits must reflect patterns of gene expression and biochemical activity unique to the hybrid(s), evidence of which would be provided by comparing the protein profiles of the hybrid(s) and the parents. While onedimensional SDS-PAGE analysis can detect alterations in expression of a number of gene products, the resolution is limited. Two-dimensional gel electrophoresis (2DGE) is increasingly used to gain new insights into plant functioning (Lery et al. 2008), as it allows the separation of several hundreds of gene products in a single gel and their subsequent identification and quantification. In recent years 2-DGE has been applied to the analysis of plant development (Hochholdinger et al. 2006, Mechin et al. 2004), deciphering of biotic or abiotic stress (Vincent et al. 2005) and characterization of plant mutants (Sorin et al. 2006).

This paper is the first report of a proteomic analysis of a somatic hybrid between Brassica oleracea and Brassica campestris, and mainly focusses on evidence of differential protein expression in the leaves of this hybrid and the parent species.

\section{Materials and Methods}

Leaves of cabbage (Brassica oleracea L. var. capitata), Chinese cabbage (B. campestris L., var. pekinensis), and a somatic hybrid (synthesized B. napus L.) variety IPSA (Institute of Postgraduate Studies in Agriculture, Bangladesh) cabbage were used as plant materials. Seeds were collected from these plants and preserved in a desiccator. Plants were grown in the greenhouse of the University of Greenwich (Medway campus), UK, the growth temperature being maintained at $22^{\circ} \mathrm{C}$. Seeds were planted in separate trays for each category. Leaves were collected from the plants from 14 days post-germination (DPG), 21DPG and 28 DPG and stored at $-80^{\circ} \mathrm{C}$ until use. 
Triplicate samples of the leaves and seeds of the somatic hybrid and its parents were frozen in liquid nitrogen and ground into a fine powder using a sterile mortar and pestle with liquid nitrogen. For each sample $100 \mathrm{mg}$ of leaf powder were then measured into a sterile Eppendorf tube and $1 \mathrm{ml}$ of ice-cold acetone containing 10\% (w/v) trichloacetic acid (TCA), 0.07\% (v/v) $\beta$-mercaptoethanol was added into the tube. The powdered samples were mixed by vortexing and then kept at $-20^{\circ} \mathrm{C}$ for $2 \mathrm{hrs}$. The extract was centrifuged at 16,000 $\mathrm{g}$ for $20 \mathrm{~min}$ at $4^{\circ} \mathrm{C}$ and the supernatant discarded. Each pellet was resuspended and washed in $1 \mathrm{ml}$ cold acetone containing $0.07 \%(\mathrm{v} / \mathrm{v}) \beta$-mercaptoethanol. The samples were vortexed and centrifuged at $16,000 \mathrm{~g}$ for $20 \mathrm{~min}$ at $4^{\circ} \mathrm{C}$ and the supernatant again discarded. Washing steps were repeated until all pellets were colourless. After discarding the final supernatant, the pellet was dried under vacuum for 2 - $5 \mathrm{~min}$. Each vacuum-dried pellet was then resuspended in $950 \mu \mathrm{l}$ freshly prepared lysis buffer $(10 \%(\mathrm{w} / \mathrm{v})$ TCA, $0.07 \%(\mathrm{v} / \mathrm{v}) \beta$-mercaptoethanol in acetone) and kept at $-20^{\circ} \mathrm{C}$ for $2 \mathrm{hrs}$. The suspensions were then sonicated on ice for 3 - 6 sessions of $15 \mathrm{sec}$ each and further incubated on a rotary shaker for 10$15 \mathrm{~min}$ at room temperature. Then $5 \mu \mathrm{l}$ of $99 \% \mathrm{~N}, \mathrm{~N}$-dimethylacrylamide (DMA) were added to each lysate followed by incubation on a rotary shaker for $30 \mathrm{~min}$ at room temperature, to effect alkylation. Excess DMA was quenched by adding $10 \mu \mathrm{l} 2 \mathrm{M}$ dithiothreitol (DTT) and insoluble material was removed by centrifugation at $16,000 \mathrm{~g}$ for $30 \mathrm{~min}$ at $4^{\circ} \mathrm{C}$. Each supernatant was stored at $-20^{\circ} \mathrm{C}$ until protein concentration determination by the Bradford method (Bradford 1976) and analysis by gel electrophoresis analysis.

For both 1D and 2D gel analyses $100 \mu \mathrm{g}$ amounts of protein were taken from each sample.

For 1D SDS PAGE analysis was carried out on NuPAGE gel (4 - 12\%) using MES buffer and then stained by simply blue safety stain (Life Technologies) following the manufacturer's instructions. The gels were scanned using the Epichemi Gel Doc system (UVP Ltd., Cambridge, UK).

For 2D gels separation in the first-dimension, by isoelectric focusing (IEF), was performed using 13-cm linear IPG strips ( $\mathrm{pH}$ 3-10) in the IPGphor system. All IPG strips were rehydrated with $250 \mu \mathrm{l}$ of dehydration buffer (8 M urea, $2 \%$ CHAPS, $0.5 \%$ ampholytes, $0.002 \%$ bromophenol blue) containing $100 \mu \mathrm{g}$ of protein. The voltage settings for IEF were $200 \mathrm{~V}$ for $20 \mathrm{~min}, 450 \mathrm{~V}$ for $15 \mathrm{~min}, 750$ $\mathrm{V}$ for $15 \mathrm{~min}$ and $2000 \mathrm{~V}$ for $30 \mathrm{~min}$. Separation in the second dimension followed immediately. The IPG gel strips were incubated in equilibration buffer 1 (50 mM Tris- $\mathrm{HCl}$ ( $\mathrm{pH} 8.8$ ), $6 \mathrm{M}$ urea, 30\% glycerol, 2\% SDS, 0.002\% bromophenol blue, $1 \%$ DTT), followed by equilibration buffer $2(50 \mathrm{mM}$ Tris- $\mathrm{HCl}, \mathrm{pH} 8.8,6 \mathrm{M}$ urea, $30 \% \mathrm{v} / \mathrm{v}$ glycerol, $2 \%$ SDS, $0.002 \%$ bromophenol blue, $2.5 \%$ iodoacetamide), for 
15 min. The strips were subsequently placed onto a 4 - 12\% IPG-well NuPAGE Tris-Bis gel $(18 \times 16 \mathrm{~cm})$ with a Tris-glycine buffer system, as described by Natarajan et al. 2005. Strips were overlayed with $0.5 \%$ agarose. The electrophoresis was performed using the XCell SureLock MinI-Cell Electrophoresis system from Life Technologhies according to the manufacturer's recommendations (Invitrogen). The 2D-PAGE gels were then stained with colloidal Coomassie Blue for $3 \mathrm{hrs,} \mathrm{destained} \mathrm{with} \mathrm{distilled} \mathrm{water,} \mathrm{and} \mathrm{finally}$ stored in $2 \% \mathrm{NaCl}$ solution at room temperature. The gels were scanned using the Epichemi Gel Doc system.

Triplicate 2-DGE analysis was performed on the protein samples from each of the three plant types. Differentially expressed protein spots from leaf proteins in 2D gels were identified by careful visual inspection on triplicate gels. There are numerous protein spots visible in the original gel and many of them are masked by the highly expressed leaf proteins. Therefore, 5 protein spots were selected which are clearly visible and differentially expressed (Fig. 2).

Protein spots were accordingly excised from the stained gel as "gel plugs", and washed with ultrapure water and then sent to Department of Biology of University of York, UK for trypsin digestion and protein identification by Mass Spectrometry. The protocols followed are described below.

In gel tryptic digestion was performed after reduction with DTE and Scarbamidomethylation with iodoacetamide. Gel pieces were washed two times with $50 \%(\mathrm{v} / \mathrm{v}$ ) aqueous acetonitrile containing $25 \mathrm{mM}$ ammonium bicarbonate, then once with acetonitrile and dried in a vacuum concentrator for $20 \mathrm{~min}$. Sequencing-grade, modified porcine trypsin (Promega) was dissolved in the 50 $\mathrm{mM}$ acetic acid supplied by the manufacturer, then diluted five-fold by adding $25 \mathrm{mM}$ ammonium bicarbonate to give a final trypsin concentration of $0.02 \mu \mathrm{g} / \mu \mathrm{l}$. Gel pieces were rehydrated by adding $10 \mu \mathrm{l}$ of trypsin solution, and after $30 \mathrm{~min}$ enough $25 \mathrm{mM}$ ammonium bicarbonate solution was added to cover the gel pieces. Digests were incubated overnight at $37^{\circ} \mathrm{C}$.

A $1 \mu \mathrm{l}$ aliquot of each peptide mixture was applied directly to the ground steel MALDI target plate, followed immediately by an equal volume of a freshlyprepared $5 \mathrm{mg} / \mathrm{ml}$ solution of 4-hydroxy- $\alpha$-cyano-cinnamic acid (Sigma) in 50\% aqueous (v/v) acetonitrile containing $0.1 \%$, trifluoroacetic acid (v/v).

Positive-ion MALDI mass spectra were obtained using a Bruker ultraflex III in reflectron mode, equipped with a Nd : YAG smart beam laser. MS spectra were acquired over a mass range of $\mathrm{m} / \mathrm{z} 800$ - 4000. Final mass spectra were externally calibrated against an adjacent spot containing 6 peptides (des-Arg1Bradykinin, 904.681; Angiotensin I, 1296.685; Glu1-fibrinopeptide B, 1750.677; ACTH (1-17 clip), 2093.086; ACTH (18-39 clip), 2465.198; ACTH (7-38 clip), 
3657.929). Monoisotopic masses were obtained using a SNAP averagine algorithm (C 4.9384, N 1.3577, O 1.4773, S 0.0417, H 7.7583) and a S/N threshold of 2 .

For each spot ten strongest peaks of interest, with an $\mathrm{S} / \mathrm{N}$ greater than 30 , were selected for MS/MS fragmentation. Fragmentation was performed in LIFT mode without the introduction of a collision gas. The default calibration was used for MS/MS spectra, which were baseline-subtracted and smoothed (Savitsky-Golay, width $0.15 \mathrm{~m} / \mathrm{z}$, cycles 4); monoisotopic peak detection used a SNAP averagine algorithm (C 4.9384, N 1.3577, O 1.4773, S 0.0417, H 7.7583) with a minimum $\mathrm{S} / \mathrm{N}$ of 6 . Bruker flexAnalysis software (version 3.3) was used to perform the spectral processing and peak list generation for both the MS and MS/MS spectra.

Tandem mass spectral data were submitted to database searching using a locally-running copy of the Mascot program (Matrix Science Ltd., version 2.1), through the Bruker Protein Scape interface (version 2.1). Search criteria included: enzyme, trypsin; fixed modifications, carbamidomethyl (C), variable modifications, oxidation (M), peptide tolerance, $250 \mathrm{ppm}, \mathrm{MS} / \mathrm{MS}$ tolerance, 0.5 Da.

Protein identification was performed by searching the National Center for Biotechnology Information (NCBI) non-redundant database using the Mascot search engine, which uses a probability-based scoring system. The following parameters were used for database searches with MALDI-TOF peptide mass fingerprinting data: monoisotopic mass, 25 ppm mass accuracy, trypsin as digesting enzyme with 1 missed cleavage allowed, carbamidomethylation of cysteine as a fixed modification, oxidation of methionine, N-terminal pyroglutamic acid from glutamic acid or glutamine as allowable variable modifications. For database searches with MS/MS spectra, the following parameters were used: average mass; 1.5 Da peptide and MS/MS mass tolerance ; peptide charge of $+1,+2$, or +3 ; trypsin as digesting enzyme with 1 missed cleavage allowed; carbamidomethylation of cysteine as a fixed modification; oxidation of methionine, N-terminal pyroglutamic acid from glutamic acid or glutamine as allowable variable modifications. Taxonomy was limited to green plants for both MALDI and MS/MS ion searches. For MALDI-TOF-MS data to qualify as a positive identification, a protein's score had to equal or exceed the minimum significant score. Positive identification of proteins by MS/MS analysis required a minimum of two unique peptides, with at least one peptide having a significant ion score. 


\section{Results and Discussion}

1D gel analysis of leaf proteins from 14, 21 and 28 DPG showed no obvious qualitative differences in protein profiles i.e. all samples shared all the bands observed in the gel (Fig. 1). However, there are two prominent bands with apparently increased amounts of protein in the hybrid, at all growth stages, relative to the parents (Fig. 1A, B), and their molecular weights of c. $52 \mathrm{kDa}$ and c. $19 \mathrm{kDa}$, indicative of the large and small subunits of ribulose-1,5-bisphosphate carboxylase (Rubisco). Leaf proteins from the cabbage (CB), Chinese cabbage (CCB) and somatic hybrid (CBS) at different growth stages were subjected to $1 \mathrm{D}$ gel analysis and at least 15 protein bands were generated from each sample (Fig 1). No significant protein profile differences between samples were observed. Two prominent bands c. $52 \mathrm{kDa}$ (marked A) and c. $19 \mathrm{kDa}$ (marked B) were seen in all samples (Fig. 1). These two bands are probably large and small subunits of rubisco (Ribulose-1,5-bisphosphate carboxylase/oxygenase) since rubisco is the most abundant protein in plant leaves, comprising approximately $50 \%$ of the total protein (Kapralov and Filatov 2007). Rubisco is a decahexameric holozenzyme consisting of eight large subunits ( $\mathrm{RbcL}$ ) and eight small subunits (RbcS), with a molecular mass of $560 \mathrm{kDa}$. RbcS is encoded by a multigene family of 2 to 22 genes, according to species, located in the nucleus, and encoding 14 - 18 $\mathrm{kDa}$ polypeptides. RbcL is specified by a single gene present in the chloroplast, encoding 45 - $55 \mathrm{kDa}$ large polypeptide (Kapralov and Filatov 2007, Ogawa et al. 2012, Warren et al. 2000). Other semi permanent bands of c. $45 \mathrm{kDa}$ and c. $26 \mathrm{kDa}$ are probably degraded large subunit proteins since degradation of rubisco is readily triggered by alterations in the rate of photosynthesis and the nitrogen economy (Zhang et al. 2012).

Due to the similar protein profiles through developmental stages 14 to 28 DPG, only protein samples from $\mathrm{CB}, \mathrm{CCB}$ and CBS at 14 DPG were subjected to 2D gel analysis.

Since leaf proteins from cabbage, Chinese cabbage and their somatic hybrid showed similar profiles on 1D gel analysis, at all three developmental stages examined, only one developmental stage, (14 DPG), was selected for 2D gel analysis. 2D gels 502, 503 and 504 represent cabbage (CB), Chinese cabbage (CCB) and their somatic hybrid (CBS), respectively (Fig. 2). There is a strong overall similarity, as expected from the $1 \mathrm{D}$ gel result, but there is substantially more complexity evident in the hybrid proteome. Spot 503-1, with a MWt of c. $52 \mathrm{kDa}$, and clearly observed in all three samples (though not labeled in gels 502 and 504), was used as an internal control. This spot, identified as the large subunit of rubisco (Table 1), is presumably a minor isoelectric form of the main 
component, which forms a massive protein spot at the same molecular weight position in all three gels, but appears most abundant in the hybrid.

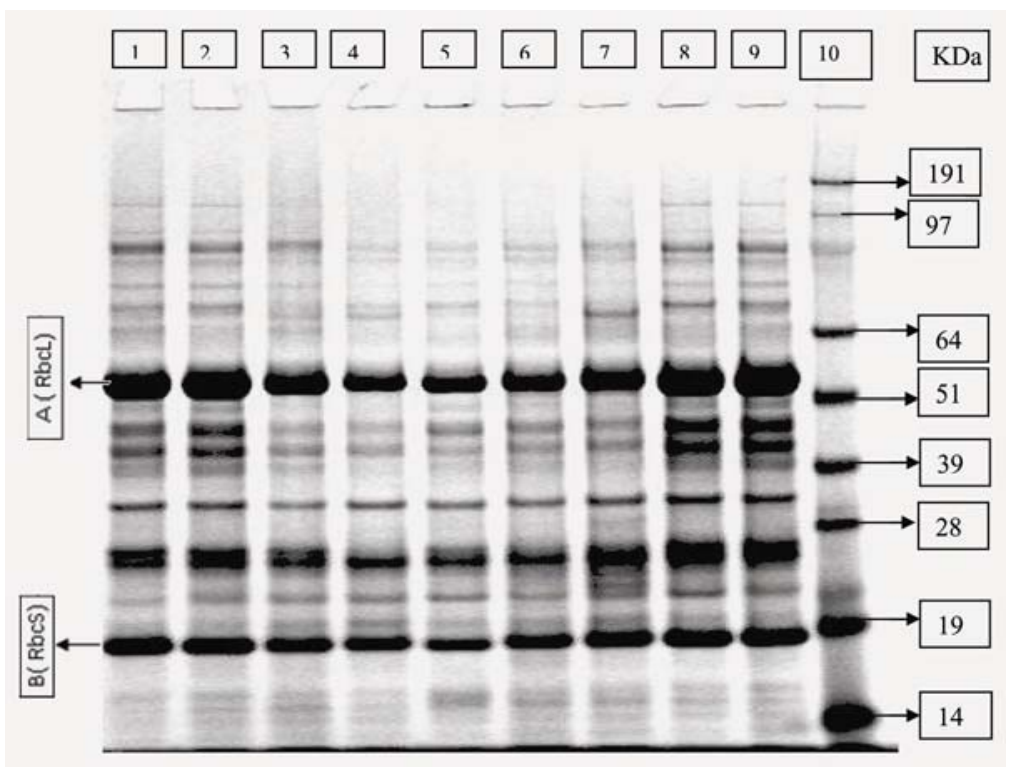

Fig. 1. 1D gel analysis of proteins of Brassica leaves collected at different growth times. Lanes: 1 - 3 leaf stage, 14 DPG; 4 - 6 leaf stage, 21 DPG; 7 - 9 leaf stage 28 DPG. Lanes 3, 4 and 7 from Chinese cabbage; 1,5 and 8 from cabbage; 2,7 and 9 from somatic hybrid. The markers used were SeeBlue Plus2 pre-stained standards (Invitrogen).

Four spots: 502-1, 502-2, 502-3 and 504-1 indicating substantially different expression levels of the relevant proteins in the three plant types, were also more closely examined. The MWt of spot 502-1 (gel 502, CB) is c. $24 \mathrm{kDa}$, and its equivalent was observed on gel 504 (CBS), with a possibly reduced level, but it was not seen in gel 503 (CCB). Spot 502-2, MWt c. $45 \mathrm{kDa}$, was seen in gel 502 (CB) but was not observed in 503 (CCB) and showed a higher level in gel 504 (CBS). Spot 502-3, MWt $19 \mathrm{kDa}$, was present in 502 (CB) and - at a higher level in 504 (CBS), but absent in gel 503 (CCB). Spot 504-1, MWt $19 \mathrm{kDa}$, was present in all samples but highest in 504 (CBS) followed by 502 (CB).

All five spots were excised and the extracted proteins analyzed via trypsinisation, MS and Mascot searching: the putative identities of the protein spots (Table 1). 
Hossain et al.

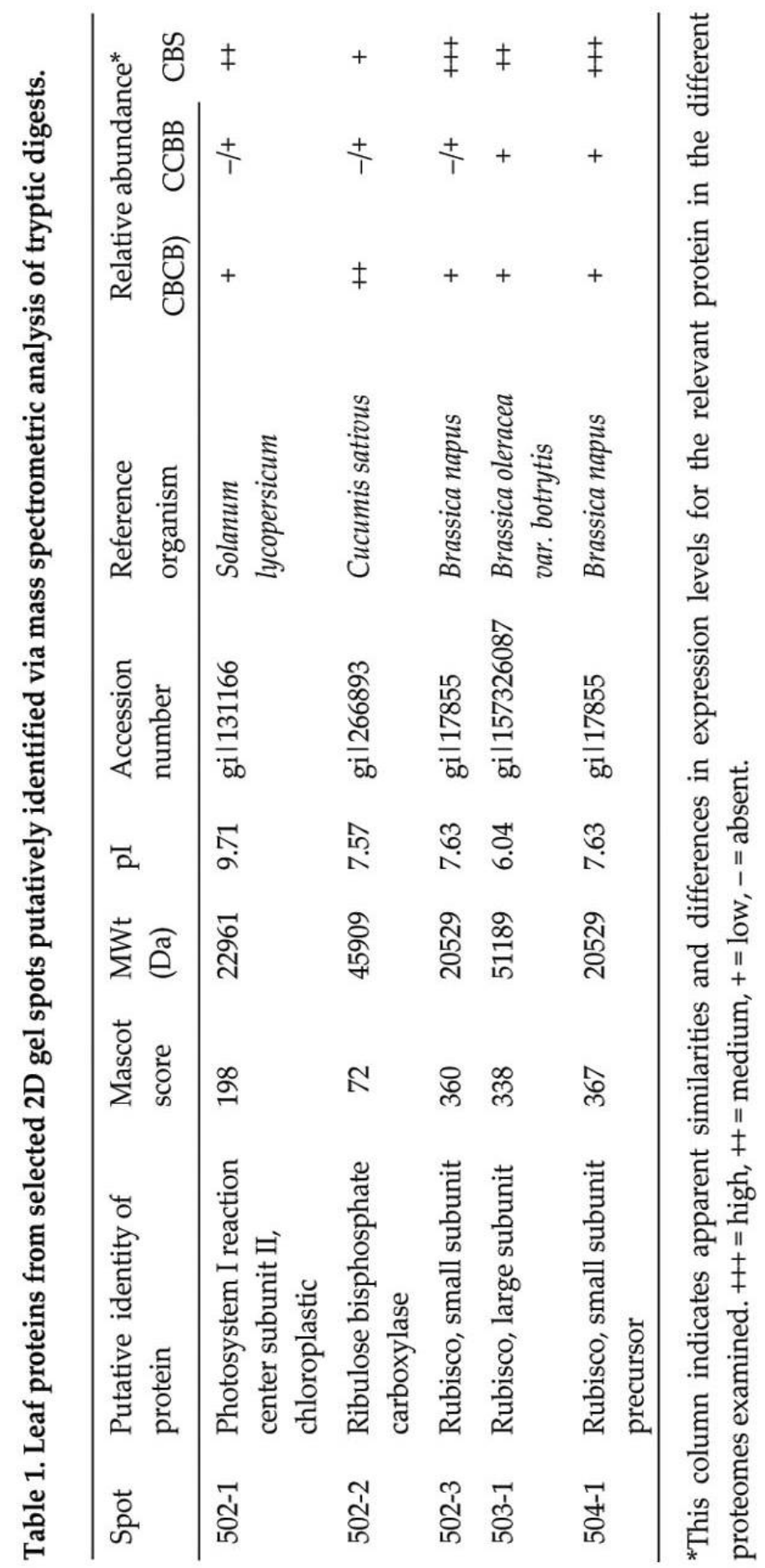


Five protein spots, including the standard, appeared differentially expressed to various degrees on gels 502 (CB), 503 (CCB) and 504 (CBS) (Table 1), and were selected for protein identification. All five spots were identified as photosynthesis-related proteins e.g. rubisco (must be acknowledged that 2D gel analysis of low abundance proteins is much hindered by rubisco in nearly all species (Krishnan and Nataranjan 2009). The identities and relative abundances of these proteins are summarized in Table 1.

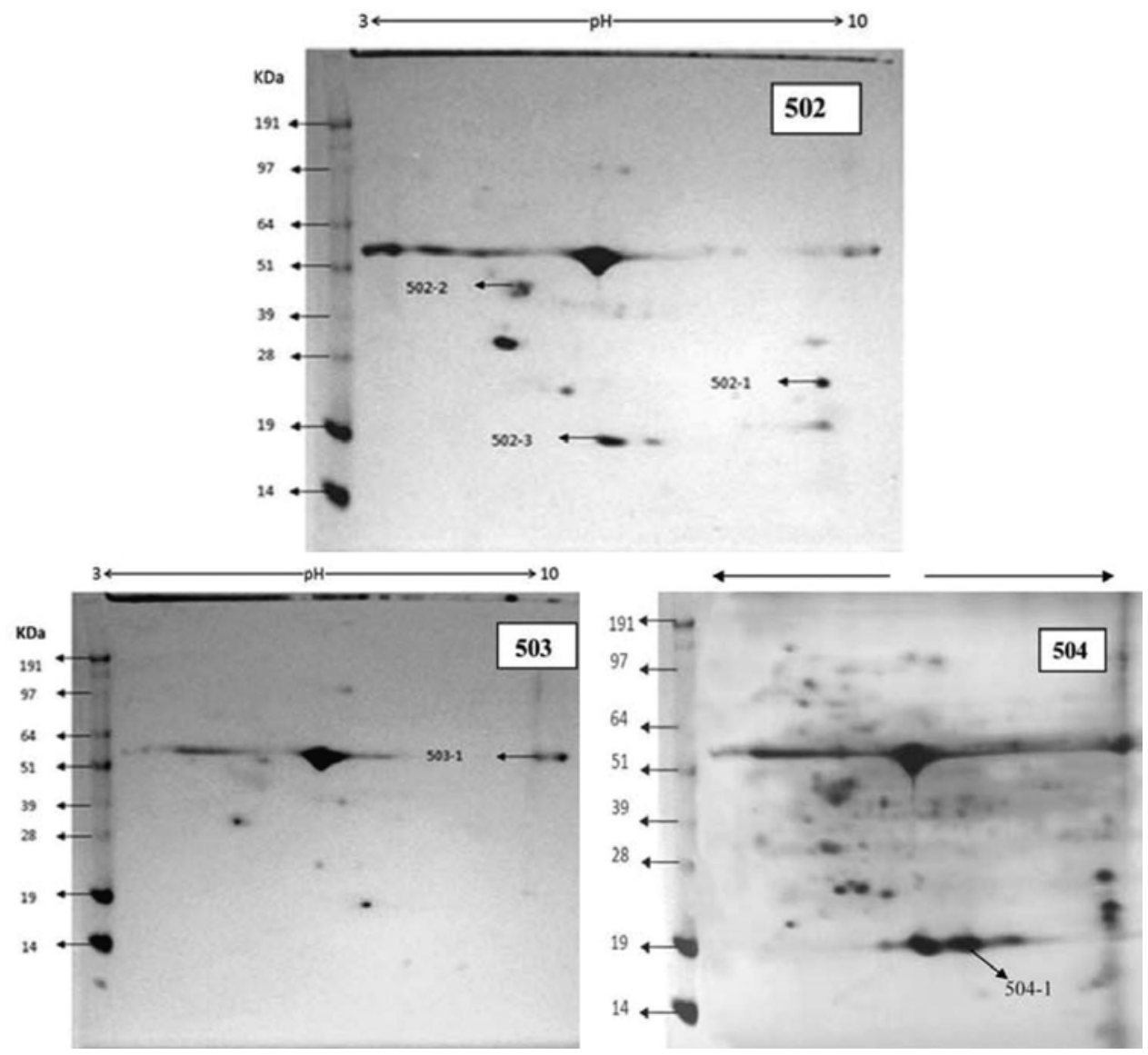

Fig. 2. 2D gel analysis of proteins from leaf of cabbage (502), Chinese cabbage (503) and somatic hybrid (504). The spot numbers of representative proteins were excised for protein identification and relative expression levels are indicated in Table 1.

Spot 502-1, identified as chloroplastic photosystem I reaction center subunit II, appeared to be more highly expressed in the hybrid than the cabbage, and at a much lower in the Chinese cabbage. Spot 502-2, identified as the large subunit of rubisco, RbcL was evident in cabbage, but found at a lower level in the hybrid and much lower in Chinese cabbage. Spot 502-3 was identified as a precursor of 
the small subunit of rubisco, RbcS, and was seen in the cabbage, with a very low level in the Chinese cabbage, but the highest levels were in the hybrid. Spot 503-1 was identified as the large subunit of rubisco, $\mathrm{RbcL}$, and was more abundant in the hybrid than the parents. Like 502-3, spot 504-1 was identified as RbcS precursor; it was found to be the highest in the hybrid, followed by cabbage and Chinese cabbage. These two RbcS precursor spots, with different pIs are apparently different forms of RbcS, perhaps reflecting multiple RbcS genes (Koivu 2004).

Overall, this 2D analysis indicates that the somatic hybrid produces more $\mathrm{RbcS}$ and $\mathrm{RbcL}$ subunits than its parents, cabbage and Chinese cabbage, and this may enhance the production of rubisco: rbcs regulates rubisco formation via activation of transcription or translation of the rbcL subunit, thus raised RbcS levels should increase levels of rbcL subunits, and thence the levels of the rubisco holoenzyme (rodermel 1999). It has been reported that RbcS, not RbcL, controls the formation of Rubisco, and a positive correlation has been observed between rubisco content and the total RbcS mRNA (Cohen et al. 2006). It has also been suggested that a balanced expression of the respective RbcS genes is important for accumulation of Rubisco holoenzyme in rice (Ogawa et al. 2012). The authors therefore speculate that accumulation of more Rubisco in the somatic hybrid, because of up-regulation of the RbcS protein, can contribute to a faster growth rate (Parikh et al. 2006) in the hybrid. This vigour characteristic may also be seen in the hybrid's large seed mass (on average, $5 \mathrm{mg}$ compared to 3.4 and $2 \mathrm{mg}$ in $\mathrm{CB}$ and $\mathrm{CCB}$, respectively) and early germination. It has been observed that seeds with greater mass produce more vigorous plants (Gonzalez 1993). Elevated photosystem I subunit levels in the hybrid could also be a factor in hybrid growth vigor.

\section{Acknowledgement}

The authors would like to thank the Commonwealth Scholarship Commission in the UK for providing a Fellowship (BDCF-2009-15) to M. M. Hossain to carry out this work.

\section{References}

Bradford MM (1976) A rapid and sensitive method for the quantification of microgram quantities of protein utilizing the principle of protein-dye binding. Anal. Biochem. 72: 248-254.

Choung PV, Deslauriers C, Kott L and Beversdorf WD (1988) Effects of donor genotype and bud sampling on microspore culture of Brassica napus. Can. J. Bot. 66(8): 16531657. 
Cohen I, Sapir Y and Shapira M (2006) A conserved mechanism controls translation of Rubisco large subunit in different photosynthetic organisms. Plant Physiol. 141: 10891097.

Gonzalez EJ (1993) Effect of seed size on germination and seedling vigor of Virola koschuyi Warb. Forest Ecol. Management 57: 275-281.

Hochholdinger F, Sauer M, Dembinsky D, Hoecker N, Muthreich N, Saleem M and Liu Y (2006) Proteomic dissection of plant development. Proteomics 6: 4076-4083.

Hossain MM, Inden $\mathbf{H}$ and Asahira T (1988) Intergeneric and interspecific hybrids through in vitro ovule culture in the Cruciferae. Plant Sci. 58: 121-128.

Hossain MM and Asahira T (1992) Development of heat tolerant somatic hybrids by PEG-mediated protoplasts fusion between Brassica oleracea L. and B. campestric L. Plant Tiss. Cult. 2: 61-69.

Hossain MM and Yazawa S (1994) High frequency plant generation from fused protoplast between B. oleracea L. and B. campestris L. Plant Tiss. Cult. 4: 93-103.

Inomata N (1997) Wide hybridization and meiotic pairing. In: Recent advances in oil seed Brassica, Kalia HR and Gupta SK (eds.), Kalyani Publications, India, New Delhi, Madras, pp. 53-76.

Kapralov MV and Filatov DA (2007) Widespread positive selection in the photosynthetic Rubisco enzyme. BMC Evol. Biol. 7: 73-82.

Krishnan HB nd Natarajan SS (2009) A rapid method for depletion of rubisco from Soybean (Glycine max) leaf for proteomic analysis of lower abundance proteins, Phytochem. 70: 1958-1964.

Koivu K (2004) Novel sprouting technology for recombinant protein production, In: Molecular farming- Plant-made pharmaceuticals and technical proteins, Fischer $\mathrm{R}$ and S. Schillberg S (Eds.) Wiley-VCH. pp. 37-53.

Lery LMS, VonKruger WMA, Viana FC, Teixeira KRS and Bisch PM (2008) A comparative proteomic analysis of Gluconacebacter diazotrophicus PAL5 at exponential and stationary phases of cultures in the presence of high and low levels of inorganic nitrogen compound. Biochem. Biophys. Acta 1784: 1578-1589.

Mechin V, Balliou T, Chateau-Joubert S, Davanture M, Olivier L, Negroni L, Prioul J-L, Thevenot C, Zivy M and Demerval C (2004) A two-dimensional proteome map of maize endosperm. Phytochem. 65: 1609-1618.

Nataranjan S, Xu C, Caperna TJ and Garett WM (2005) Comparison of protein solubilization methods suitable for proteomic analysis of soybean seed proteins. Anal. Biochem. 342: 214-220.

Ogawa S, Suzuki Y, Yoshizawa R, Kanno K and Makino A (2012) Effect of individual suppression of RBCS multigene family on rubisco contents in rice leaves. Plant Cell Environ. 35: 546-563.

Parikh MR, Greene DN, Woods KK and Matsumara I (2006) Directed evolution of rubisco hymorphs through genetic selection in engineered E. coli. Protein Eng. Design Select. 19: 113-119.

Rodermel S (1999) Subunit control of rubisco biosynthesis - a relic of an endosymbiotic past? Photosynthesis Res. 59: 105-123. 
Sorin C, Negroni L, Balliau T, Corti H, Jacquemot M-P, Davanture M, Sanberg G, Zivy $\mathbf{M}$ and Bellini C (2006) Proteomic analysis of different mutant genotypes of Arabidopsis led to the identification of 11 proteins correlating with adventitious root development. Plant Physiol. 140: 349-364.

Vincent D, Lapierre C, Pollet B, Cornic G, Negroni L and Zivy M (2005) Water deficit affect caffeate o-Methyltransferase, lignifications, and related enzymes in maize leaves. A proteomic investigation. Plant Physiol. 137: 949-960.

Warren CR, Adams MA and Chen Z (2000) Effect of N-source on concentration of rubisco in Eucalyptus diversicolor, as measured by capillary electrophoresis. Physiologia Plantarum. 110: 52-58.

Yamagishi H, Hossain MM and Yonezawa K (1992) Production of somatic hybrids between southern type Chinese cabbage 'Kenshin' and cabbage 'Yoshin' and their flowering characteristics. J. Japan. Soc. Hort. Sci. 61: 311-316.

Zhang L, Zhang Y, Rui Q, Ren $\mathbf{Y}$ and Xu L (2012) Effects of ATP and Zn 2+ in degradation of the large subunit of ribulose-1,5-biphosphate carboxylase/oxygenase in wheat leaves. African J. Agri. Res. 7 : 711-720. 